Series A

I. MATHEMATICA

378

\title{
ON HOMOLOGY THEORIES IN LOCALLY CONNECTED SPACES II
}

BY

OLLI JUSSILA

H E L S I N K I 1965

S U O M A L A N EN TIEDEAKATEMIA 
Communicated 9 April 1965 by P. J. Myrberg and Oldi Lehto 


\section{On homology theories in locally connected spaces II}

Introduction. Our purpose is to prove the theorems of the first paper of this series [6] for Borel homology ([1]), for the homology theories of Deheuvels ([2]) and for the homology of residue complexes in noetherian preschemes with finitely generated coefficient sheaves ([4]). Steenrod homology is included as a special case of Borel homology. In [6] these theorems were already proved for singular and Alexander-Kolmogoroff homologies ([6]), pp. 6, 7, 12, 14), since the latter is isomorphic to the projective homology of a grating spectrum (see [7], p. 279). Note that in these theorems the homology groups $H_{n}(N)$ are canonically isomorphic to the corresponding Čech homology groups of $X$, if each open covering of $X$ has a refinement in the net $N$ and vice versa.

We recall for convenience some definitions in [6]. Let $X$ be a topological space and $C: 0 \leftarrow C_{-1} \leftarrow C_{0} \leftarrow C_{1} \leftarrow \ldots$ a complex on $X$ ([6], p. 3). In the terminology of Deheuvels ([2]) $C$ is an antisheaf of complexes on the ordered set of subsets of $X$. Let $N$ be a net of coverings of $X$ ([6], p. 5). Each $\alpha \in N$ defines a double complex $\stackrel{\alpha}{C}_{*}$ and ${ }^{\prime} \stackrel{\alpha}{C}_{* q}(q>-2)$ is by definition the augmented complex $C_{*}\left(\alpha, C_{q}\right)$ of chains of the nerve of a with coefficients in the antisheaf $C_{q}([6])$, p. 4). All homology theories in question can be obtained from complexes of this kind and, if there is a coefficient group, $C_{-1}$ can be chosen as the constant antisheaf associated with it. To get what we want we need only to prove that

$$
H_{p}\left({ }^{\prime} C_{* q}\right)=H_{p}\left(C_{*}\left(\alpha, C_{q}\right)\right)=0
$$

for each $\alpha \in N, p>-2, q>-1$ (see [6], p. 5). In particular, the homology of $C_{*}$ will then be canonically isomorphic to the corresponding Cech homology in paracompact, $l c_{n-1}$ and semi- $l c_{n}$ spaces (relative to $C_{*}$ ).

1. The Borel homology theory of locally compact spaces can be defined as follows: Let $K$ be a Dedekind ring and $K^{*}$ its quotient field. Let $X$ be a locally compact space and $\tilde{C}^{*}: 0 \rightarrow \tilde{C}^{0} \rightarrow \tilde{C}^{1} \rightarrow \ldots$ the standard injective resolution of the constant sheaf $K$ on $X$ ([1], p. 138). Denote by $C^{*}: 0 \rightarrow C^{0} \rightarrow C^{1} \rightarrow \ldots$ the presheaf of cochain complexes on the ordered 
set of subsets of $X$ obtained from $\tilde{C}^{*}$ by applying the section functor $\Gamma_{c}$. The dual $D_{*}$ of $C^{*}$ is a complex on $X$ defined as follows: For each $P \subset X, D_{*}(P)=D\left(C^{*}(P)\right) \quad$ (see $[1]$, p. 142). By definition $D_{n}(P)=$ $\operatorname{Hom}_{K}\left(C^{n}(P), K^{*}\right) \oplus \operatorname{Hom}_{K}\left(C^{n+1}(P), K^{*} / K\right)(n>-1)$ and $D_{-1}(P)=$ $\operatorname{Hom}_{K}\left(C^{0}(P), K^{*} / K\right)$. The functorial maps $C^{*}(P) \rightarrow C^{*}(Q)(Q \subset P \subset X)$ induce by transposition the functorial maps $d(P, Q): D_{*}(P) \leftarrow D_{*}(Q)$. $\tilde{C}^{*}$ is c-soft. Hence the homology groups of $D_{*}(P)$ are canonically isomorphic to the Borel homology groups $H_{n}(P, K)$ for each compact $P \subset X$. Beacause $K^{*}$ and $K^{*} / K$ are injective and $\tilde{C}^{*}$ is c-soft, the functorial maps $d(P, Q)$ are monomorphisms allowing $D_{*}(Q)$ to be considered as a subcomplex of $D_{*}(P)$ for all compact sets $Q \subset \mathrm{P}$.

The modules $C^{n}(P)$ ( $P$ compact) are divisible as quotients of the divisible module $C^{n}(X)$, hence the modules $D_{n}(P) \quad(P$ compact $)$ are $K$-flat and torsion free. If $B$ is a $K$-module, it follows that for all compact $Q \subset P, D_{*}(Q) \otimes_{K} B$ can be considered as a subcomplex of $D_{*}(P) \otimes_{K} B$. For each $U \subset X$ denote by $D_{*}^{C}(U)$ the direct limit (or union) of the complexes $D_{*}(P)\left(P \subset U\right.$ compact). Then $D_{*}^{C}$ and $D_{*}^{C} \otimes_{K} B$ are complexes on $X$. We claim that they satisfy $(0.1)$ for any open covering $\alpha$ of $X$ and all values of $p$ and $q$ greater than -2 . This is true for $D_{*}$ and for any finite family $\beta$ of compact subsets of $X$, because

$$
\begin{gathered}
H_{p}\left(C^{*}\left(\beta, D_{q}\right)\right)=H_{p}\left(\operatorname{Hom}_{K}\left(C^{*}\left(\beta, C^{q}\right), K^{*}\right)\right) \oplus H_{p}\left(\operatorname{Hom}_{K}\left(C^{*}\left(\beta, C^{q+1}\right), K^{*} / K\right)\right) \\
=\operatorname{Hom}_{K}\left(H^{p}\left(C^{*}\left(\beta, C^{q}\right)\right), K^{*}\right) \oplus \operatorname{Hom}_{K}\left(H^{p}\left(C^{*}\left(\beta, C^{q+1}\right)\right), K^{*} / K\right)=0(q>-1) \\
\text { and } \mathrm{H}_{p}\left(C_{*}\left(\beta, D_{-1}\right)\right)=\operatorname{Hom}_{K}\left(H^{p}\left(C^{*}\left(\beta, C^{0}\right)\right), K^{*} / K\right)=0 .
\end{gathered}
$$

The same conclusion follows for $D_{*} \otimes_{K} B$ from the universal coefficient theorem. If now $\alpha=\left(U_{i}\right)_{i \in I}$ is any open covering of $X$ and $c=\Sigma_{s \in I^{p+1}} c_{s} \in$ $C_{p}\left(\alpha, D_{q}^{c}\right)\left(c_{s} \in D_{q}^{c}\left(\cap_{i \in s} U_{i}\right)\right)$, there is a finite family $\beta=\left(P_{i}\right)_{i \in I^{\prime}}\left(I^{\prime} \subset I\right)$ of compact subsets of $U_{i}(i \in I)$ such that $c_{s}=0$, if $s \notin I^{\prime p+1}$ and $c_{s} \in$ $D_{q}\left(\cap_{i \in s} P_{i}\right)$, if $s \in I^{\prime p+1}$. This means that $c \in C_{p}\left(\beta, D_{q}\right) \subset C_{p}\left(\alpha, D_{q}^{c}\right)$. If $c$ is a cycle, it is a boundary in $C_{*}\left(\beta, D_{q}\right)$ and à fortiori in $C_{*}\left(\alpha, D_{q}^{c}\right)$. The same conclusion follows for $D_{*}^{c} \otimes_{K} B$ by tensoring the modules above with $B$, and we have proved (0.1) in the case of Borel homology.

If we denote by $C_{0}$ the kernel of $D_{0}^{c} \rightarrow D_{-1}^{c}$, the Borel homology groups $H_{n}^{c}(U, K)$ (U open in $X, n>-1$ ) can be computed from the complex

$$
0 \leftarrow C_{0}(U) \leftarrow D_{1}^{c}(U) \leftarrow \ldots
$$

(see [1], theorems 3.4 and 3.7 and remark p. 144. Note that the homology commutes with direct limits.) If $X$ is $h c_{0}$, it is locally connected, and $H_{-1}^{c}(U, K)=0$ for each open $U$ in $X$ (see [1], pp. 149-151). Hence the sequence $0 \rightarrow C_{0} \rightarrow D_{0}^{c} \rightarrow D_{-1}^{c} \rightarrow 0$ is exact. Because $D_{0}^{c}$ and $D_{-1}^{c}$ satisfy 
(0.1), so also does $C_{0}$. By taking tensor products with $B$ one gets a similar complex for $H_{n}^{c}(U, B)$. A mapping of $X$ to a point gives a surjective augmentation of this complex by the constant antisheaf $B$ ([1], 6.1, p. 148), and the homology groups of this augmented complex are isomorphic to the corresponding augmented Borel homology groups of $U$ in dimensions greater than -1 .

Hence we get from theorem $3,[6], p .11$ the following result: If $X$ is a paracompact, locally compact, $l c_{n-1}$ and semi-lc $c_{n}$ space relative to Borel homology with compact supports, then the Borel homology groups $H_{n}^{c}$ $(X, B)$ and the Čech homology groups $\check{\check{H}}_{n}(X, B)$ and $\check{H}_{n}^{c}(X, B)(n>0)$ are canonically isomorphic. In particular, the Cech homology is exact and has compact supports.

Remark 1. The isomorphism $H_{n}^{c}(X, B) \cong \check{H}_{n}^{c}(X, B)$ remains in fact valid without the paracompactness condition (roughly speaking we need strong n-refinements only on the compact supports), although lemma 1 in [6], p. 7, must then be replaced by a more general one. Note that this isomorphism cannot be concluded directly from the same result in the compact case, because the set of compact, $l c_{n-1}$ and semi- $l c_{n}$ subsets need not be cofinal in the set of all compact subsets.

Remark 2. If $X$ is compact and $K$ is a field, the isomorphism $H_{n}(X, K) \cong \check{H}_{n}(X, K)$ is well-known without any local connectedness conditions.

2. In [2] Deheuvels has defined homology theories for presheaves and antisheaves on topological spaces as hyperderived functors of certain composed functors. They can be defined by resolving functors as follows: Let $\mathcal{D}$ be the set of open and proper coverings $\alpha$ of $X$, which are closed to the right i.e. $U \subset U_{i}, U_{i} \in \alpha \Rightarrow U \in \alpha$. If $A$ is a presheaf on $X$ and $U_{0} \supset U_{1}$ $\supset \ldots \supset U_{n}$ with $U_{i} \in \alpha$ for $i=0, \ldots, n$, set $A\left(U_{0}, \ldots, U_{n}\right)=A\left(U_{0}\right)$. Let $p_{U_{0}}^{i} \ldots U_{n}: A\left(U_{0}, \ldots, U_{n}\right) \rightarrow A\left(U_{0}, \ldots, \hat{U}_{i}, \ldots, U_{n}\right)$ be the identity $A\left(U_{0}\right) \rightarrow A\left(U_{0}\right)$ for $i>0$ and the functorial map $A\left(U_{0}\right) \rightarrow A\left(U_{1}\right)$ for $i=0$. Denote by $C_{*}(\alpha, A)$ the chain complex

$$
\begin{aligned}
& 0 \leftarrow \oplus_{U_{0} \in \alpha} A\left(U_{0}\right) \leftarrow \frac{\partial_{1}}{\longleftarrow} \oplus_{U_{1} \subset U_{0} \in \alpha} A\left(U_{0}, U_{1}\right) \leftarrow \partial_{2} \ldots . \\
& \leftarrow \stackrel{\partial_{n}}{\longleftarrow} \oplus_{U_{n} \subset U_{n-1} \subset \ldots \subset U_{0} \in \alpha} A\left(U_{0}, \ldots, U_{n}\right) \leftarrow \partial_{n+1} \ldots
\end{aligned}
$$

where $\partial_{n}=\oplus_{U_{n} \subset U_{n-1} \subset \ldots \subset U_{0} \in a}\left(\sum_{i=0}^{n}(-1)^{i} P_{U_{0} \ldots U_{n}}^{i}\right)$.

If $\beta \in \Phi$ is a refinement of $\alpha$ then $\beta \subset \alpha$ and $C_{*}(\beta, A) \subset C_{*}(\alpha, A)$. Denote by $\mathcal{K}(X)$ the double complex $\left(K(X)_{i j}\right)$ with $K(X)_{i j}=0$ for 
$i>0$ or $j<0$ and with differentials ' $d: K(X)_{i j} \rightarrow K(X)_{i-1, j}, " d: K(X)_{i j}$ $\rightarrow K(X)_{i, j-1}$, defined as follows:

$$
K(X)_{i j}=\prod C_{j, \alpha_{0}, \ldots, \alpha_{-i}} \quad(i<1),
$$

where the product ranges over all sequences $\alpha_{0} \subset \alpha_{1} \subset \ldots \subset \alpha_{-i}$ in $\mathscr{S}$ and $C_{j, \alpha_{0}, \ldots, \alpha_{-i}}=C_{j}\left(\alpha_{-i}, A\right)$. "d is induced by $\partial_{j}$ and

$$
\begin{gathered}
{ }^{\prime} d\left(a_{\left.j, \alpha_{0}, \ldots, \alpha_{-i}\right)_{\alpha_{0}} \subset \ldots \subset \alpha_{-i}=\left(b_{j}, \beta_{0}, \ldots, \beta_{-i+1}\right.}\right)_{\beta_{0}} \subset \ldots \subset \beta_{-i+1} \\
b_{j, \beta_{0}, \ldots, \beta_{-i+1}}=\sum_{k=0}^{-i+1}(-1)^{k} a_{j, \beta_{0}, \ldots, \hat{\beta}_{k}, \ldots, \beta_{-i+1}}
\end{gathered}
$$

$\left(C_{j, \beta_{0}, \ldots, \hat{\beta}_{k}, \ldots, \beta-i+1} \subset C_{j, \beta_{0}, \ldots, \beta_{-i+1}}\right)$. The corrected Čech homology groups $\breve{H}_{n}(X, A)$ are the homology groups of $\mathcal{K}(X)$ endowed with the total differential and grading. Note that these homology groups can be non-trivial in all negative dimensions.

If $U$ is an open subset of $X$ and $\alpha \in \mathscr{D}$, we denote by $\alpha \mid U$ the covering of $U$ consisting of elements of $a$ contained in $U$. Obviously $\alpha \backslash U \subset$ $\alpha \mid V$, if $U \subset V$. If we replace the groups $C_{j, \alpha_{0}, \ldots, \alpha_{-i}}$ by the corresponding groups $C_{j, \alpha_{0}\left|U, \ldots, \alpha_{-i}\right| U}$ in the definitions, we get a double complex $\mathcal{K}(U)=\left(K(U)_{i j}\right)$. If $U \subset V$, we have a natural inclusion $\mathcal{K}(U) \subset$ K $(V)$ induced by the inclusions $\alpha_{k}\left|U \subset \alpha_{k}\right| V(k=0, \ldots,-i)$. Hence the functor $\mathcal{K}: U \rightarrow \mathcal{K}(U)$ is a complex on the open subsets of $X$ and defines there a homology theory $H$. If $\alpha$ is any open covering of $X$, we can suppose without changing the homology that all elements of $\mathscr{L}$ are refinements of $\alpha$. Then each $\mathcal{K}_{j}: U \rightarrow \mathcal{K}(U)_{j}$ is isomorphic to a direct product of direct sums of coelementary antisheaves $A_{U_{0}}\left(U_{0} \subset U_{i}, U_{i} \in \alpha\right)$ such that

$$
A_{U_{0}}(U)=A\left(U_{0}\right) \text {, if } U_{0} \subset U \text {, and } 0 \text { otherwise, }
$$

the functorial map $A_{U_{0}}(U) \rightarrow A_{U_{0}}(V)$ is the identity, if either

$$
U_{0} \subset U \text { or } U_{0} \notin V \text {, and zero, if } U_{0} \notin U \text {. }
$$

To verify $(0.1)$ it is therefore sufficient to show that $H_{i}\left(\alpha, A_{U_{0}}\right)=0$ fcr all values of $i$. The elements of $\alpha$ containing $U_{0}$ form a closed non-empty subcomplex $\Delta$ of the nerve of $\alpha$. Clearly $H_{i}\left(\alpha, A_{U_{0}}\right)=H_{i}\left(\Delta, A_{U_{0}}\right)$ for all $i$. The assertion follows because the coefficient system $A_{U_{0}}$ is constant on $\Delta$ and $\Delta$ as a star is homotopically trivial.

If the coefficient presheaf $A$ is constant and $H_{-1}(\mathcal{K}(U))=0$ for each open $U$ in $X$, we can augment the complex

$$
0 \leftarrow \mathcal{K}_{0} \leftarrow \mathcal{K}_{1} \leftarrow \ldots
$$


by $A$ as we did in the previous section. Otherwise it can be augmented for example by Coker $\left(\mathcal{K}_{1} \leftarrow \mathcal{K}_{0}\right)$.

All these results can be obtained for antisheaves simply by replacing all sequences $U_{0} \subset \ldots \subset U_{n}$ by the opposite sequences $U_{0} \supset \ldots \supset U_{n}$.

The same argument as used in [6], p. 12, for singular homology again shows the validity of theorems 1 and 3 .

Remark. In [2], pp. 316-317 Deheuvels defined for any antisheaf $B$ the homology groups $H_{n}(X, B)$ as hyperderived functors of a composed functor $\Gamma_{D *}\left\{L_{R}, X\right\}_{R \in D}$. In the category of modules, in which we are operating, these groups are trivial in connected spaces, if the closed points are dense and non-isolated. This can be seen as follows: Any antisheaf $B$ has a projective resolution $९$ of the form

$$
0 \leftarrow \oplus_{U \subset X} C_{U}^{0} \leftarrow \oplus_{U \subset X} C_{U}^{1} \leftarrow \ldots \ldots
$$

(see [2], p. 265), where each $C_{U}^{i}$ is a coelementary antisheaf associated with $U$ (see definition in the previous section). From the definition it follows that $\left(\oplus_{U \subset X} C_{U}^{i}\right)(V)=\oplus_{U \subset V} C_{U}^{i}(U)$, the functorial maps $\left(\oplus_{U \subset X} C_{U}^{i}\right)(V) \rightarrow$ $\left(\oplus_{U \subset X} C_{U}^{i}\right)(W)(V \subset W \subset X)$ being inclusions. If $x \in X$, then $\Gamma_{x}\left(\oplus_{U \subset X} C_{U}^{i}\right)$ $=\lim _{x \in V}\left(\oplus_{U \subset V} C_{U}^{i}(U)\right)=\bigcap_{x \in V}\left(\oplus_{U \subset V} C_{U}^{i}(U)\right)$, which is zero unless $x$ has a minimal neighborhood $U_{0}$. If closed points are dense, we must have $U_{0}=\{x\}$ i.e. $x$ must be isolated, which is contrary to our assumption. If $g$ is an injective resolution of the presheaf $\left\{L_{R, X} P B\right\}_{R \in D}$ on $\mathscr{D}^{*}$, then the double complex $\left.K \Gamma_{I^{*}} \cdot 9 L_{R, x} P B\right\}$ is trivial. Since its homology groups are isomorphic to the groups $H_{n}(X, B)$, we have proved our result.

3. Let $\left(X, \circlearrowleft_{X}\right)$ be a noetherian prescheme and let $X_{p}(p>-1)$ be the set of $p$-dimensional points of $X$. For each $x \in X_{p}$ let $M_{x}$ be the (constant) sheaf on $\{x\}$ associated with an $\mathscr{O}_{x}$-module $D_{x}([4], p .113)$ and let $i^{x}, X$ denote the inclusion of $\{x\}$ in $X$. Then the residue complex of $X$ is a chain complex of $\mathscr{C}_{X}$-modules $\mathcal{K}_{X}=\left(\mathcal{K}_{X, p}\right)_{p>-1}$. where $\mathcal{K}_{X, p}$ is the direct sum (over $X_{p}$ ) of the sheaves $i_{*}^{x, X}\left(M_{x}\right)$. $\mathcal{K}_{X, p}$ is generated by the presheaf $K_{p}: U \rightarrow \oplus_{x \in X_{p}} \Gamma\left(i_{*}^{x}, X_{(}\left(M_{x}\right) \mid U\right)=\oplus_{x \in U_{p}} D_{x}$. We show that this presheaf is already a sheaf i.e. satisfies the conditions $F I$ and FII in [3], p. 109. Let $\alpha=\left(U_{i}\right)_{i \in I}$ be a family of open subsets of $X$ and let $U$ be their union. $F I$ is clear because each $x \in U_{p}$ is contained in some $U_{i}$. Let $\left(s_{i}=\right.$ $\left.\Sigma_{x_{i, j}} m_{i, j}\right)_{i \in I}\left(x_{i, j} \in\left(U_{i}\right)_{p}, m_{i, j} \in D_{x_{i, j}}\right)$ be a family of elements of $K_{p}\left(U_{i}\right)$ $(i \in I)$ such that the restrictions of $s_{i}$ and $s_{k}$ coincide on $U_{i} \cap U_{k}(i, k \in I)$ i.e. $m_{i, j}=m_{k, n}$ whenever $x_{i, j}=x_{k, n}$. There is obviously a family $\left(m_{x}\right)_{x \in X_{p}}$ of elements of $D_{x}\left(x \in X_{p}\right)$ such that $m_{x}=m_{i, j}$ whenever $x=$ $x_{i, j}$. To prove $F I I$ it is sufficient to note that only a finite number of the $m_{x}: \mathrm{s}$ are non-zero, because $U$ is quasicompact. The natural map $K_{p} \rightarrow$ 
$\mathcal{K}_{X, p}$ is thus an isomorphism. Each inclusion $U \subset V \subset X$ of open subsets induces natural isomorphisms $\mathcal{K}_{U, p} \cong \mathcal{K}_{V, p} \mid U$. The corresponding inclusions $U_{p} \subset V_{p}$ induce a natural map $\mathcal{K}_{U} \rightarrow \mathcal{K}_{V}$. The induced maps $\Gamma\left(\mathcal{K}_{U, p}\right) \rightarrow \Gamma\left(\mathcal{K}_{V, p}\right)$, i.e. $K_{p}(U) \rightarrow K_{p}(V)$, can be identified with the inclusions $\oplus_{x \in U_{p}} D_{x} \rightarrow \oplus_{x \in V_{p}} D_{x}$.

If $F$ is an $\mathscr{C}_{X}$-module of finite type, we define $C_{*}(X, F)=$ Hom $_{O_{X}}$ $\left(X ; F, \mathcal{K}_{X}\right)$. For each inclusion $U \subset V \subset X$ of open sets the maps $F \mid V \rightarrow$ $F \mid U$ and $\mathcal{K}_{U} \rightarrow \mathcal{K}_{V}$ induce a functorial map $C_{*}(U, F \mid U) \rightarrow C_{*}(V, F \mid V)$. The inclusion maps $h_{x}: i_{*}^{x, X}\left(M_{x}\right) \rightarrow \mathcal{K}_{X, p}$ induce a map $h: \oplus_{x \in X_{p}}$ Hom $_{\mathcal{O}_{X}}$ $\left(X ; F, i_{*}^{x}, X_{x}\left(M_{x}\right)\right) \rightarrow \operatorname{Hom}_{\mathcal{O}_{X}}\left(X ; F, K_{X, p}\right)$. We claim that $h$ is an isomorphism. Each $f_{x} \in \operatorname{Hom}_{O_{X}}\left(X ; F, i_{*}^{x, X}\left(M_{x}\right)\right)$ is a family of maps $f_{x}(U): F(U)$ $\rightarrow i_{*}^{x, X}\left(M_{x}\right)(U)$ compatible with the restriction maps and $h\left(\Sigma_{x \in X_{p}} f_{x}\right)(U)$ is the map $\oplus_{x \in X_{p}} f_{x}(U): F(U) \rightarrow\left(\oplus_{x \in X_{p}} i_{*}^{x, X}\left(M_{x}\right)\right)(U)=\oplus_{x \in X_{p}} i_{*}^{x}, X\left(M_{x}\right)$ $(U)$. The injectivity of $h$ is clear. To prove the surjectivity let $f$ be an element of $\operatorname{Hom}_{O_{X}}\left(X ; F, \mathcal{K}_{X, p}\right)$ i.e. a family of maps $f(U): F(U) \rightarrow$ $\left(\oplus_{x \in X_{p}} i_{*}^{x, X}\left(M_{x}\right)\right)(U)=\oplus_{x \in X_{p}} i_{*}^{x, X}\left(M_{x}\right)(U)$ satisfying the obvious compatibility conditions. For each $x \in X_{p}$ and $U \subset X$ denote by $f_{x}(U)$ the map which associates with each $a \in F(U)$ the component of $F(U)(a)$ associated with $x$. Each family $\left(f_{x}(U)\right)_{U \subset X}$ defines an element $f_{x}$ of $\operatorname{Hom}_{\mathcal{O}_{X}}(X ; F$, $\left.i_{*}^{x, X}\left(M_{x}\right)\right)$. Because $F$ is of finite type and $X$ is quasicompact, $f$ is completely determined by its values on a finite number of sections (see [5], p. 46). It follows that $f_{x}$ is non-zero only on a finite number of $p$-dimensional points $x$ of $X$. Obviously $h\left(\Sigma f_{x}\right)=f$, which proves the surjectivity of $h$.

$\operatorname{Hom}_{O_{X}}\left(X ; F, i_{*}^{x, X}\left(M_{x}\right)\right)$ is canonically isomorphic to $\operatorname{Hom}_{\mathcal{O}_{x}}\left(F_{x}, D_{x}\right)$ ([5], p. 42, $(4.4,3.1))$, and the map $C_{p}(U, F) \rightarrow C_{p}(V, F)$ can be identified with the natural inclusion $\oplus_{x \in U_{p}} \operatorname{Hom}_{\mathcal{O}_{x}}\left(F_{x}, D_{x}\right) \rightarrow \oplus_{x \in V_{p}} \operatorname{Hom}_{\mathcal{O}_{x}}\left(F_{x}, D_{x}\right)$. Let $C=\left(C_{p}\right)_{p>-1}$ be the complex on $X$ defined by

$$
C_{p}(U)=C_{p}(U, F)(p>-1) .
$$

The argument used in [6], p. 6, for the singular complex again shows the validity of (0.1) for $C$ and for the net of open and proper coverings of $X$. Hence different augmentations of $C$ give rise to different homology theories, which can be compared with the corresponding $\check{C}$ ech homology theories by means of theorem 1, [6], p. 8.

4. To show that the paracompactness condition is indispensable in theorem 3, part I §4, we exhibit below a locally contractible Hausdorff space, whose singular homology is different from the corresponding Čech homology: Let $S^{2}$ be a two-sphere, $A$ and $B$ two points on $S^{2}$ and let $l$ be a geodesic joining $A$ to $B$ ( $A$ and $B$ are not included in $l$ ). We define a topology $T$ 
on $S^{2}$ as follows: $A$ has a fundamental system of neighborhoods obtained by omitting all points of $l$ from the members of a converging sequence of metric neighborhoods of $A$, which do not contain $B$. Elsewhere $T$ is supposed to coincide with the standard metric topology of $S^{2}$. Endowed with this topology $S^{2}$ is neither regular not paracompact and it has the properties mentioned above. The open subsets $U=S^{2}-A, V=S^{2}-l-B$ cover $S^{2}$ and it is easy to verify that $U, V$ and $U \cap V$ are contractible. The triviality of the singular homology groups of $S^{2}$ follows from the exactness of the Mayer-Vietoris sequence of the open triad $(U, V, \underset{v}{U} \cap V)$. On the other hand it is easy to check that the two-dimensional Cech homology group of $S^{2}$ is isomorphic to the coefficient group in contradiction with the claim of theorem 3. The above argument is valid for the homology of any fine complex (with respect to finite open coverings), which satisfies the homotopy axiom. In fact, the exact Mayer-Vietoris sequence can be easily derived from the spectral sequence of the covering $\{U, V\}$ in the homology theory in question. We leave the details to the reader.

The author gratefully acknowledges the support of the Institute for Advanced Study during the preparation of this paper.

The Institute for Advanced Study, Princeton, N.J. 


\section{References}

[1] Borel, A., Moore, J.C. Homology theory for locally compact spaces - Michigan Math. J. 7 (1960), 137-159.

[2] Deheuvels, R. Homologie des ensembles ordonnés - Bull. Soc. Math. France 90 (1962), $261-321$.

[3] Godement, R. Théorie des faisceaux. - Actualités Sci. Ind. 1251, Hermann, Paris, 1958.

[4] Grothendieck, A. Cohomology theory of abstract algebraic varieties - Proc. Intern. Congress of Math., 103-118, Edinburgh (1958).

[5] Éléments de Géométrie Algébrique, I, I.H.E.S. - Publications Mathématiques, No $4,1960$.

[6] Jussila, O. On homology theories in locally connected spaces - Ann. Acad. Sci. Fenn. A.I., 340 (1964).

[7] Lefschetz, S. Algebraic topology - Amer. Math. Soc. Colloquium publications (1942).

[8] Steenrod, N. Regular cycles of compact metric spaces - Ann. of Math. (2), 41 (1940), 833-851. 